\title{
Nouveaux Travaux Pratiques d'Elaboration et de Caractérisation de cellules de mémoires résistives de type OxRAM
}

\author{
Marceline Bonvalot ${ }^{1,2}$, Delphine Constantin ${ }^{2}$, John Pointet ${ }^{2}$, Ahmad Bsiesy ${ }^{1,2}$ \\ ${ }^{1}$ Univ. Grenoble Alpes, CNRS, LTM, F-38000, Grenoble, France \\ ${ }^{2}$ Centre Interuniversitaire de Microélectronique et Nanotechnologies (CIME Nanotech), \\ Contact email : marceline.bonvalot@cea.fr
}

\begin{abstract}
Cet article présente la mise en œuvre d'une nouvelle formation pratique pour la réalisation et la caractérisation électrique de cellules mémoires basées sur la commutation réversible entre deux états de résistance distincts dans le matériau diélectrique d'une structure MIM. L'enchaînement des étapes technologiques s'appuie sur les techniques élémentaires traditionnelles en microélectronique, et permet de donner une vue globale des problématiques actuelles dans le domaine de la fabrication des composants.
\end{abstract}

\section{Introduction}

Le CIME Nanotech, Centre Interuniversitaire de Microélectronique et Nanotechnologies, pôle CNFM de Grenoble, s'emploie à répondre aux évolutions de besoins en formation expérimentale en micro et nanoélectronique en développant son offre en travaux pratiques. Fort de son partenariat double avec les filières de formation et les laboratoires de recherche, le CIME Nanotech est capable d'introduire des nouveaux sujets de formation sur des thématiques récemment issues du monde de la recherche et qui sont en cours de transfert au monde industriel. Ainsi, les étudiants se trouvent en contact avec les nouvelles technologies et peuvent mesurer de façon concrète tous les enjeux de la conception et la fabrication de circuits intégrés.

Dans ce contexte, le CIME Nanotech a enrichi son catalogue de formations en septembre 2016 en proposant de nouveaux travaux pratiques sur la fabrication et la caractérisation de mémoires résistives ReRAMs (Resistive Random Acess Memory) qui sont en passe de remplacer les mémoires FLASH pour certaines applications, notamment dans le domaine des mémoires embarquées.

\section{Contexte scientifique}

Le domaine des mémoires intégrées non volatiles connaît actuellement une transformation profonde imposée par la miniaturisation des composants, par la réduction de la puissance électrique consommée et par des temps de lecture et d'écriture toujours plus faibles. A ce titre, les mémoires résistives ReRAM suscitent d'importants efforts de recherches à l'heure actuelle, car elles sont considérées comme le candidat idéal pour remplacer les mémoires non volatiles actuelles de type FLASH. En effet, elles présentent des possibilités de miniaturisation attrayantes aux nœuds inférieurs à $20 \mathrm{~nm}$, ainsi qu'une consommation énergétique moindre et une vitesse de commutation élevée (1).

Ces mémoires résistives sont constituées de deux électrodes métalliques séparées par un matériau isolant pour former une structure MIM (Métal Isolant Métal). Le changement de résistance de la structure induit par l'application d'une tension détermine l'état OFF (forte résistance) ou l'état $\mathrm{ON}$ (basse résistance) de la mémoire. Plusieurs mécanismes physiques 
peuvent être à l'origine de cette variation de résistance dans le diélectrique, ce qui permet de distinguer plusieurs classes de mémoires résistives. Par exemple, lorsque le changement de résistance est engendré par un changement de phase du matériau isolant (cristallin $\rightarrow$ amorphe ou amorphe $\rightarrow$ cristallin), lui-même induit par un cycle de chauffage/refroidissement par effet Joule sous l'application d'une tension, les mémoires correspondantes sont appelées mémoires à changement de phase PCRAM (Phase Change RAM) (2). Dans les mémoires dites OxRAM, le matériau isolant de la structure MIM est un oxyde métallique, typiquement $\mathrm{HfO}_{2}$. Le passage d'un état de forte résistance vers un état de faible résistance dans cet oxyde est provoqué par la création puis la migration de lacunes d'oxygène sous l'action d'une contrainte électrique. Le champ électrique entraîne la rupture de liaisons Métal-Oxygène dans l'oxyde métallique, ce qui engendre des lacunes d'oxygène $\left(\mathrm{VO}_{2}{ }^{+}\right)$qui migrent de la cathode vers l'anode jusqu'à établir un chemin de conduction entre les deux électrodes métalliques ; la structure MIM est alors dans un état de faible résistance. L'application d'une tension de polarité inverse conduit à la rupture de ce filament conducteur par la contre-diffusion des lacunes d'oxygène qui peuvent éventuellement se recombiner localement avec des ions $\mathrm{O}_{2}{ }^{-}$au voisinage de l'anode. Il est ainsi possible d'écrire et d'effacer la mémoire grâce à deux seuils de tension caractéristiques (Figure 1).

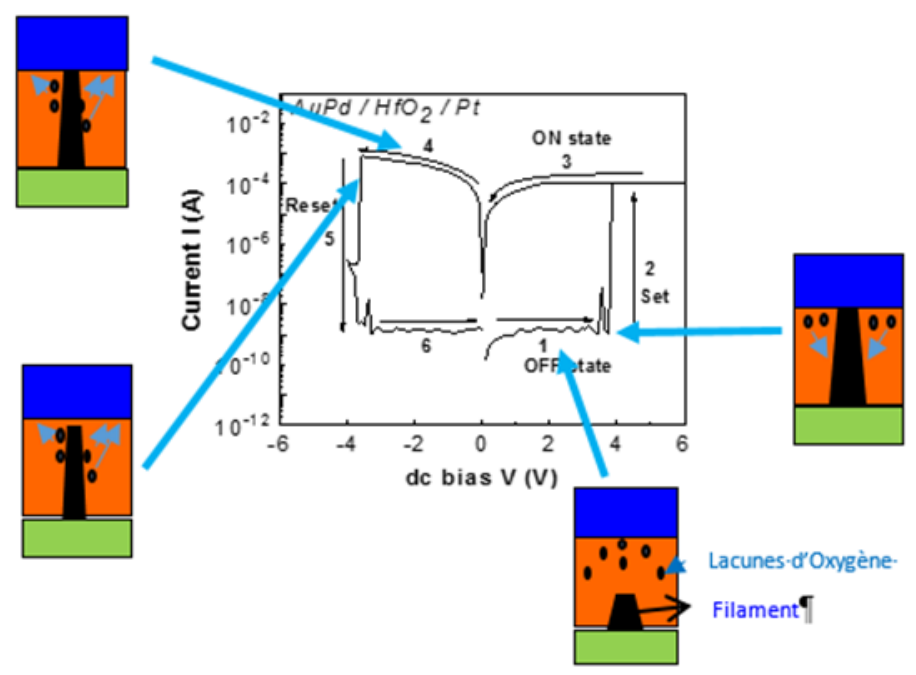

Fig.1. Cycle d'hystérésis montrant les phases d'écriture et d'effacement d'une cellule OxRAM.

\section{Programme pédagogique}

La formation que nous avons mise en place au CIME Nanotech propose de réaliser des cellules résistives de type OxRAM et de les caractériser électriquement pour étudier leur comportement mémoire. Elle se déroule au sein de deux plateformes expérimentales du CIME Nanotech, la salle blanche et la caractérisation électrique.

\section{Elaboration de cellules en Salle blanche}

Pour cela, nous avons défini un procédé qui repose sur l'enchaînement de plusieurs étapes technologiques élémentaires (Figure 2) et qui se déroule sur une séance pédagogique d'une journée de travail ( 8 heures) en salle blanche. 


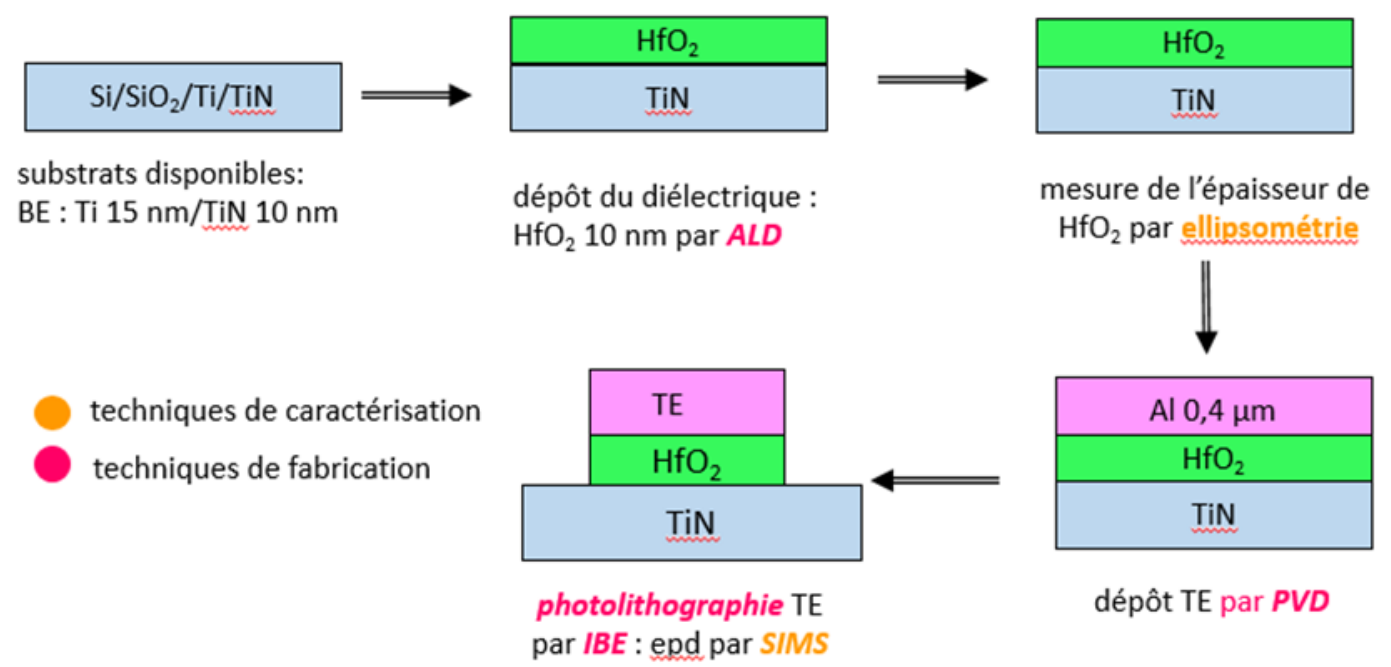

Fig.2. Enchaînement des étapes technologiques mises en œuvre pour la fabrication de cellules OxRAM.

En amont de la séance pédagogique, les substrats sont recouverts d'une couche de TiN, matériau de l'électrode inférieure (bottom electrode BE), déposée en pleine plaque. La séance pédagogique débute par le dépôt d'une couche mince d'oxyde, typiquement $10 \mathrm{~nm}$ de $\mathrm{HfO}_{2}$, par Atomic Layer Deposition (ALD). Cette technique, relativement récente dans l'industrie, est l'occasion d'aborder les mécanismes fondamentaux mis en jeu au cours de la croissance par voie chimique (CVD) sur des substrats activés thermiquement (nucléation, adsorption vs absorption) et la limitation inhérente de «conformalité » de la couche mince obtenue dans les structures à fort rapport d'aspect par exemple. L'examen de la recette de dépôt ALD permet d'expliquer la croissance d'épaisseur contrôlée qui s'appuie sur l'introduction séquentielle des précurseurs pour fabriquer le matériau monocouche par monocouche grâce aux réactions chimiques auto-limitantes à la surface du substrat. Une mesure par ellipsométrie de l'épaisseur du dépôt $\mathrm{HfO}_{2} \mathrm{ALD}$ permet a posteriori de vérifier que cette valeur est bien conforme à l'épaisseur visée au cours du dépôt, déterminée par le nombre de cycles défini dans la recette.

L'étape technologique suivante est le dépôt d'une couche de métal (Al $0.4 \mu \mathrm{m})$ pour l'élaboration de l'électrode métallique supérieure (top électrode TE). Réalisé par PVD magnétron, ce dépôt permet aux étudiants de mettre en pratique leurs connaissances théoriques dans le domaine des plasmas et des techniques du vide tout en complétant leurs connaissances sur les techniques de dépôts par voie physique usuelles en microélectronique.

Des cellules mémoire individuelles et de plusieurs dimensions sont ensuite définies par une étape de lithographie optique traditionnelle suivie d'une gravure réalisée sous faisceau d'ions énergétiques. La description du principe de fonctionnement de cette technique de gravure est l'occasion de rappeler les principes et avantages d'une gravure sèche au regard d'une gravure humide ; le suivi de la gravure se fait grâce à un spectromètre de masse qui permet dans le cas de matériaux multicouches l'identification du matériau gravé et une détection de fin d'attaque (End Point Detection, EPD) en temps réel.

Des mesures de profondeur de couches gravées sont ensuite effectuées par profilométrie sur les cellules ainsi obtenues pour vérifier la cohérence de l'ensemble des paramètres expérimentaux accessibles à chaque étape technique : l'épaisseur de l'électrode supérieure peut être estimée à partir de la durée de l'étape de gravure par faisceau d'ions, ainsi que par la mesure de la hauteur de marche entre une cellule et l'électrode inférieure 
obtenue par profilométrie. Ces deux mesures doivent être cohérentes avec l'épaisseur visée de la couche d'aluminium déposée par PVD, qui dépend à son tour des paramètres de fonctionnement du bâti de PVD.

\section{Caractérisation électrique}

La séance d'élaboration des cellules mémoires OxRAM en salle blanche est suivie par une séance de caractérisation électrique de quatre heures sur un banc de test électrique composé d'une station sous pointe Cascade Microtech et d'un un appareil d'analyse de paramètres de semiconducteurs HP 4155a. Un cycle de polarisation électrique est appliqué au dispositif MIM en connectant l'électrode de TiN à la masse comme le montre l'encart de la figure 2. L'application de ce cycle de polarisation $0 \rightarrow 8 \rightarrow-10 \rightarrow 0$ fait une boucle d'hystérésis qui résulte d'un passage forte résistance/basse résistance (étape « set ») pour une tension positive suivi d'un passage inverse de faible résistance/forte résistance (étape « reseté ») pour une tension négative comme le montre la figure 3. On obtient ainsi une différence de quatre à cinq ordres de grandeur entre les courants des états faible résistance et forte résistance. Les étudiants peuvent grâce à l'analyse de cette caractéristique se familiariser avec le concept de mémoire résistives qui sont basée non pas sur le stockage d'une charge dans une grille flottante d'une cellule dérivées d'un transistor MOS mais sur le changement d'état de résistance d'un diélectrique. Cette analyse est également l'occasion de poser la question de la fiabilité et de l'endurance de tels composants et de comparer leurs performances par rapport aux autres technologies, notamment la technologie FLASH.

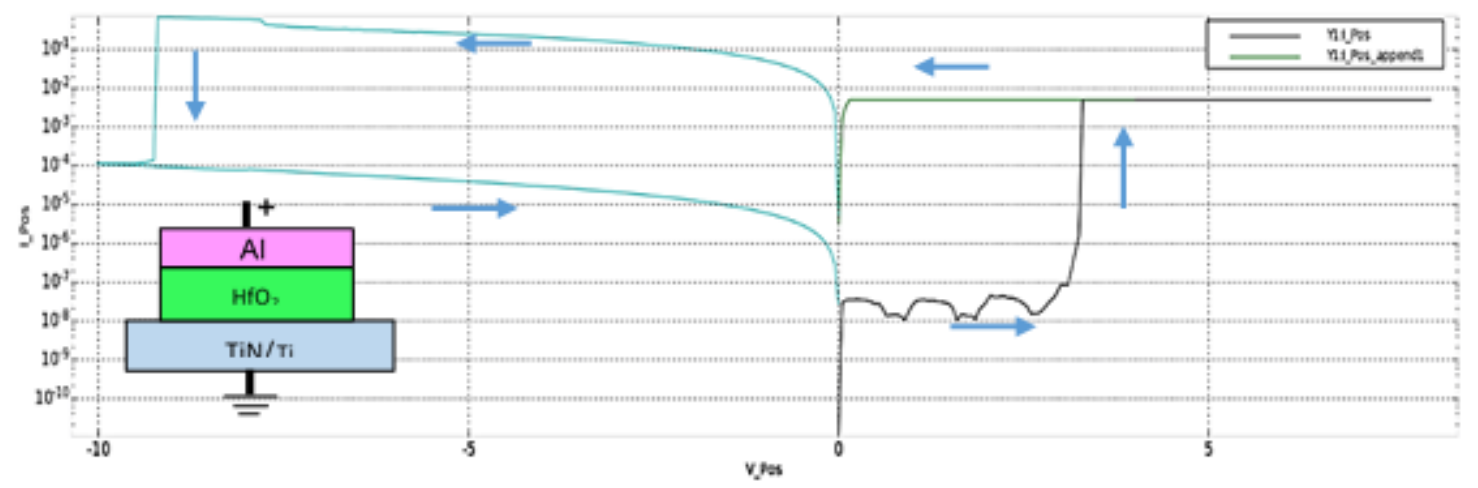

Fig.3. Cycle d'hystérésis courant-tension obtenu lors de la caractérisation d'une structure MIM de type OxRAM. L'encart de cette figure décrit le sens de la polarisation appliquée.

\section{Conclusion}

Cette formation originale sur la fabrication et la caractérisation de cellules mémoires permet de donner une vue d'ensemble des étapes front-end mises en œuvre au cours de la fabrication de dispositifs intégrés en salle blanche. Elle permet également de souligner les problématiques scientifiques actuelles soulevées à chacune de ces étapes fondamentales. De ce fait, elle répond pleinement aux objectifs pédagogiques définis au CIME Nanotech de faire coller son catalogue de formations au plus près des innovations technologiques et des recherches en cours, afin de systématiquement proposer une offre de pointe à l'ensemble de ses utilisateurs. 


\section{Remerciements}

Ce projet a été soutenu par le groupement d'intérêt public « coordination nationale pour la microélectronique et les nanotechnologies », GIP CNFM (3), via le programme ANR11-IDFI-0017_FINMINA (4) et par l'IRT Nanoélectronique ANR-10-AIRT-05 ainsi que le LabEx Minos ANR-10-LABX-55-01.

\section{Références}

1. A. Sawa : Materials Today, 2008 (11), 6 pp28-36.

2. W. Welnic, M. Wuttig : Materials Today, 2008 (11), 6 pp20-27.

3. GIP-CNFM: Groupement d'Intérêt Public - Coordination Nationale pour la formation en Microélectronique et en nanotechnologies. Website: http://wwww.cnfm.fr

4. IDEFI-FINMINA : Initiative d'Excellence - Formation Innovante en MIcroélectronique et Nanotechnologies, ANR-11-IDFI-0017. Website: http://www.cnfm.fr/VersionFrancaise/actualites/ FINMINA.htm 